PROCEEDINGS OF THE

AMERICAN MATHEMATICAL SOCIETY

Volume 126, Number 1, January 1998, Pages 225-229

S 0002-9939(98)03888-X

\title{
OPTIMAL ESTIMATION OF SHELL THICKNESS IN CUTLAND'S CONSTRUCTION OF WIENER MEASURE
}

\author{
BANG-HE LI AND YA-QING LI
}

(Communicated by Andreas R. Blass)

\begin{abstract}
In Cutland's construction of Wiener measure, he used the product of Gaussian measures on ${ }^{*} R^{N}$, where $N$ is an infinite integer. It is mentioned by Cutland and $\mathrm{Ng}$ that for the product measure $\gamma$,

$$
\gamma\left(\left\{x: R_{1} \leq\|x\| \leq R_{2}\right\}\right) \simeq 1
$$

where $R_{1}=1-(\log N)^{\frac{1}{2}} N^{-\frac{1}{2}}$ and $R_{2}=1+M N^{-\frac{1}{2}}$ with $M$ any positive infinite number. We prove here that $R_{1}$ may be replaced by $1-m N^{-\frac{1}{2}}$ with $m$ any positive infinite number. This is the optimal estimation for the shell thickness. It is also proved that $\gamma(\{x:\|x\|<1\}) \simeq \gamma(\{x:\|x\|>1\}) \simeq \frac{1}{2}$. And for the *Lebesgue measure $\mu, \mu(\{x:\|x\| \leq r\})$ is finite and not infinitesimal iff $r=(2 \pi e)^{-\frac{1}{2}} N^{\frac{1}{2}\left(1+\frac{1}{N}\right)} e^{\frac{a}{N}}$ with $a$ finite, while for the *Lebesgue area of the sphere $S^{N-1}(r), r$ should be $(2 \pi e)^{-\frac{1}{2}} N^{\frac{1}{2}} e^{\frac{a}{N}}$.
\end{abstract}

N. Cutland constructed the Wiener measure in [1] via the internal measure $\gamma$ in a nonstandard $*$-finite Euclidean space ${ }^{*} R^{N}$, where $N$ is an infinite positive integer, and

$$
\gamma(A)=\left(2 \pi N^{-1}\right)^{-\frac{1}{2} N} \int_{A} \exp \left(-\frac{1}{2} N \sum_{i=1}^{N} x_{i}^{2}\right) d x_{1} \cdots d x_{N} .
$$

$\gamma$ has a very interesting property that almost all points in ${ }^{*} R^{N}$ are near the unit sphere. So it is interesting to estimate the exact thickness of the shell with almost all points.

In Remark 2 of [2] N. Cutland and S.-A. $\mathrm{Ng}$ mentioned the following:

Let $R_{1}=1-(\log N)^{\frac{1}{2}} N^{-\frac{1}{2}}$ and $R_{2}=1+M N^{-\frac{1}{2}}$, where $M$ is any positive infinite number. Then

$$
\gamma\left(\left\{x: R_{1} \leq\|x\| \leq R_{2}\right\}\right) \simeq 1 \text {. }
$$

Actually they proved in the preprint of [2] that $R_{1}$ or $R_{2}$ cannot be replaced by $R_{1}=1-m N^{-\frac{1}{2}}$ or $R_{2}=1+m N^{-\frac{1}{2}}$, if $m$ is finite. So their estimation for the outer one is already optimal. Is their result for the inner one optimal? They said in the preprint: "we are less sure". This is indeed not optimal and the optimal estimation is shown by the following theorem.

Received by the editors July 14, 1995 and, in revised form, April 9, 1996.

1991 Mathematics Subject Classification. Primary 03H05, 28E05, 51M05; Secondary 28A35, $28 \mathrm{C} 20$.

Key words and phrases. Shell thickness, Wiener measure, *-finite Euclidean space.

This project was supported by the National Natural Science Foundation of China.

(C) 1998 American Mathematical Society 
Theorem 1. Let $R_{1}=1-M N^{\frac{1}{2}}$ and $R_{2}=1+M^{\prime} N^{\frac{1}{2}}$ where $M$ and $M^{\prime}$ are any positive infinite numbers. Then

$$
\gamma\left(\left\{x: R_{1} \leq\|x\| \leq R_{2}\right\}\right) \simeq 1 .
$$

Proof. By the results of the preprint of [2], we need only prove that

$$
\gamma\left(\left\{x:\|x\| \leq R_{1}\right\}\right) \simeq 0 .
$$

Also from the preprint of [2],

$$
\gamma(\{x:\|x\| \leq a\})=\alpha \pi^{-\frac{1}{2}} \int_{0}^{a} \beta(r) d r
$$

where $\alpha \simeq 1$ and

$$
\beta(r)=N^{\frac{1}{2}} \exp \left(\frac{1}{2} N\left(1-r^{2}\right)\right) r^{N-1}
$$

We have for $r \neq 0$ :

$$
\begin{aligned}
\beta(r) & =N^{\frac{1}{2}} \exp \left(\frac{1}{2} N\left(1-r^{2}\right)+(N-1) \log r\right) \\
& =N^{\frac{1}{2}} \exp \left(\frac{1}{2} N\left(1-r^{2}\right)+(N-1)\left(r-1-\frac{(r-1)^{2}}{2}+\varepsilon\right)\right)
\end{aligned}
$$

where $\varepsilon=\frac{(r-1)^{3}}{3(1+\xi)^{3}}$ for some $\xi$ in between $r-1$ and 0 according to the Lagrange remainder theorem for the Taylor expansion. Simplifying this gives

$$
\beta(r)=N^{\frac{1}{2}} \exp \left(-\frac{2 N-1}{2}(1-r)^{2}+\varepsilon^{\prime}\right)
$$

where $\varepsilon^{\prime}=1-r+(N-1) \varepsilon$.

Now consider $r=1-m N^{-\frac{1}{2}}$ with $m^{3} N^{-\frac{1}{2}} \simeq 0$. Then

$$
(N-1) \varepsilon=(N-1) \frac{(r-1)^{3}}{3(1+\xi)^{3}}=\frac{-(N-1)}{3(1+\xi)^{3}} m^{3} N^{-\frac{3}{2}} \simeq 0
$$

and

$$
\varepsilon^{\prime} \simeq 0
$$

Hence

$$
\beta(r)=a(r) \exp \left(\frac{1}{2} \log N-\frac{2 N-1}{2}(1-r)^{2}\right)
$$

where $a(r) \simeq 1$ for such $r$. Notice that $m$ may be negative for $(*)$ true. This will be used in the proof of Theorem 2. Let

$$
m_{0}=\left(\frac{N \log N}{2 N-1}\right)^{\frac{1}{2}}
$$

then $m_{0}$ is infinite and $m_{0}^{3} N^{-\frac{1}{2}} \simeq 0$. So the formula $(*)$ is used to give

$$
\beta\left(r_{1}\right)=a\left(r_{1}\right) \simeq 1
$$

where $r_{1}$ stands for $1-m_{0} N^{-\frac{1}{2}}$.

It is easy to check that $\beta(r) \simeq 0$ for $r>0$ with ${ }^{\circ} r<1$. Then using the Robinson Lemma there is $r_{0} \simeq 1$ such that $\beta\left(r_{0}\right) \simeq 0$ for all $0 \leq r \leq r_{0}$; and we may take 
$r_{0}<r_{1}$. Then, since $\beta(r)$ is increasing for $r<\left(1-N^{-1}\right)^{\frac{1}{2}}=r_{2}$, say, and $r_{1}<r_{2}$, we have $\beta(r) \leq 2$ for $r \leq r_{1}$ and so

$$
\int_{r_{0}}^{r_{1}} \beta(r) d r<2\left(r_{1}-r_{0}\right) \simeq 0
$$

and so

$$
\int_{0}^{r_{1}} \beta(r) d r \simeq 0
$$

Now, letting $m<m_{0}$ be any positive infinite number, we have

$$
\begin{aligned}
& \left(\int_{1-m_{0} N^{-\frac{1}{2}}}^{1-m N^{-\frac{1}{2}}} \beta(r) d r\right)^{2}=\left(\int_{1-m_{0} N^{-\frac{1}{2}}}^{1-m^{-\frac{1}{2}}} a(r) N^{\frac{1}{2}} \exp \left(-\frac{2 N-1}{2}\left(1-r^{2}\right)\right) d r\right)^{2} \\
& \quad \leq 4\left(\int_{m N^{-\frac{1}{2}}}^{m_{0} N^{-\frac{1}{2}}} N^{\frac{1}{2}} \exp \left(-\frac{2 N-1}{2} s^{2}\right) d s\right)^{2} \\
& \quad=4 N \int_{m N^{-\frac{1}{2}}}^{m_{0} N^{-\frac{1}{2}}} d s_{2} \int_{m N^{-\frac{1}{2}}}^{m_{0} N^{-\frac{1}{2}}} \exp \left(-\frac{2 N-1}{2}\left(s_{1}^{2}+s_{2}^{2}\right)\right) d s_{1} \\
& \quad \leq 4 N \int_{0}^{\frac{\pi}{2}} d \theta \int_{m N^{-\frac{1}{2}}}^{\sqrt{2} m_{0} N^{-\frac{1}{2}}} \exp \left(-\frac{2 N-1}{2} t^{2}\right) t d t \\
& =2 \pi N(2 N-1)^{-1}\left(\exp \left(-(2 N-1) N^{-1} m^{2}\right)-\exp \left(-(2 N-1) N^{-1} m_{0}^{2}\right)\right)
\end{aligned}
$$

Since $m$ and $m_{0}$ are both infinite, we conclude that

$$
\int_{1-m_{0} N^{-\frac{1}{2}}}^{1-m N^{-\frac{1}{2}}} \beta(r) d r \simeq 0 ;
$$

therefore

$$
\int_{0}^{1-m N^{-\frac{1}{2}}} \beta(r) d r \simeq 0
$$

and the theorem is proved.

It is interesting to see how much mass lies inside the unit sphere. We have

Theorem 2. $\gamma(\{x:\|x\|<1\}) \simeq \gamma(\{x:\|x\|>1\}) \simeq \frac{1}{2}$.

Proof. Let $m$ be any positive infinite number with $m^{3} N^{-\frac{1}{2}} \simeq 0$. We know from the preprint of [2] or Theorem 1 that

$$
\gamma(\{x:\|x\|>1\}) \simeq \gamma\left(\left\{x: 1+m N^{-\frac{1}{2}}>\|x\|>1\right\}\right) .
$$

Thus from the formula $(*)$ above, we have for $1<r<1+m N^{-\frac{1}{2}}$,

$$
\beta(r)=a(r) N^{\frac{1}{2}} \exp \left(-\frac{2 N-1}{2}(1-r)^{2}\right)
$$


with $a(r) \simeq 1$. Let

$$
\begin{aligned}
I & =\int_{1}^{1+m N^{-\frac{1}{2}}} N^{\frac{1}{2}} \exp \left(-\frac{2 N-1}{2}(1-r)^{2}\right) d r \\
& =\int_{0}^{m N^{-\frac{1}{2}}} N^{\frac{1}{2}} \exp \left(-\frac{2 N-1}{2} s^{2}\right) d s
\end{aligned}
$$

then

$$
\begin{aligned}
& N \int_{0}^{\frac{\pi}{2}} d \theta \int_{0}^{m N^{-\frac{1}{2}}} \exp \left(-\frac{2 N-1}{2} t^{2}\right) t d t \leq I^{2} \\
& \quad \leq N \int_{0}^{\frac{\pi}{2}} d \theta \int_{0}^{\sqrt{2} m N^{-\frac{1}{2}}} \exp \left(-\frac{2 N-1}{2} t^{2}\right) t d t .
\end{aligned}
$$

Now for any infinite positive number $M$,

$$
\begin{aligned}
& N \frac{\pi}{2} \int_{0}^{M N^{-\frac{1}{2}}} \exp \left(-\frac{2 N-1}{2} t^{2}\right) t d t \\
& \quad=\frac{\pi N}{2(2 N-1)}\left(1-\exp \left(-\frac{2 N-1}{2 N} M^{2}\right)\right) \simeq \frac{\pi}{4} .
\end{aligned}
$$

So

$$
I \simeq \frac{\sqrt{\pi}}{2}
$$

and

$$
\gamma\left(\left\{x: 1+m N^{-\frac{1}{2}}>\|x\|>1\right\}\right)=\alpha \pi^{-\frac{1}{2}} \int_{1}^{1+m N^{-\frac{1}{2}}} \beta(r) d r \simeq \frac{1}{2} .
$$

This proves the theorem.

It seems strange that the Gaussian measure $\mathcal{N}\left(0, N^{-1}\right)$ on any axis of ${ }^{*} R^{N}$ concentrates in the monad of the zero, hence in a set with ${ }^{*}$ Lebesgue measure infinitesimal, while the product measure $\gamma$ concentrates on a set with distance to the origin nearly 1 . The following theorem may give a partial explanation of this phenomenon, since it tells us that there is a ball in ${ }^{*} R^{N}$ of infinite radius with *Lebesgue measure infinitesimal.

Theorem 3. For the ${ }^{*}$ Lebesgue measure $\mu$ on ${ }^{*} R^{N}$ and surface measure $\mu_{s}$ on spheres, we have

$$
\begin{gathered}
\mu(\{x:\|x\| \leq r\}) \text { is finite and } \neq 0 \\
\text { iff } \\
r=(2 \pi e)^{-\frac{1}{2}} N^{\frac{1}{2}\left(1+\frac{1}{N}\right)} e^{\frac{a}{N}} \text { with a finite }
\end{gathered}
$$

and

$$
\begin{aligned}
& \mu_{s}(\{x:\|x\|=r\}) \text { is finite and } \nsucceq 0 \\
& \text { iff } \\
& r=(2 \pi e)^{-\frac{1}{2}} N^{\frac{1}{2}} e^{\frac{a}{N}} \text { with a finite. }
\end{aligned}
$$


Hint to the Proof. Consider the case when $N$ is even; then

$$
\mu(\{x:|x| \leq r\})=\frac{\pi^{\frac{N}{2}} r^{N}}{\left(\frac{N}{2}\right) !} \triangleq A
$$

and

$$
\ln r=\frac{1}{N} \ln \left(\frac{N}{2}\right) !-\frac{1}{2} \ln \pi+\frac{1}{N} \ln A .
$$

By Stirling's formula

$$
\left(\frac{N}{2}\right) !=(\pi N)^{\frac{1}{2}}\left(\frac{N}{2 e}\right)^{\frac{N}{2}} e^{b} \text { with } b \simeq 0
$$

we have

$$
r=(2 \pi e)^{-\frac{1}{2}} N^{\frac{1}{2}\left(1+\frac{1}{N}\right)} e^{\frac{1}{N} \ln A+\frac{b}{N}+\frac{1}{2 N} \ln \pi} .
$$

Let

$$
a=\ln A+b+\frac{1}{2} \ln \pi
$$

It is easy to see that $A>0$ is finite and $\not 40$ iff $a$ is finite. The proof for the other cases are similar.

We give some comments on the results. First, the set of $r$ 's making the balls finite and $\nsucceq 0$ is disjoint with that for the spheres. Secondly, any point in one set differs from any point in another one by an infinitesimal.

It is interesting to notice that the sets

$$
\left\{(2 \pi e)^{-\frac{1}{2}} N^{\frac{1}{2}\left(1+\frac{1}{N}\right)} e^{\frac{a}{N}} \text { with } a \text { finite }\right\}
$$

and

$$
\left\{(2 \pi e)^{-\frac{1}{2}} N^{\frac{1}{2}} e^{\frac{a}{N}} \text { with } a \text { finite }\right\}
$$

are just two different $N^{-\frac{1}{2}} O$-equivalence classes according to the language in [3], and the set

$$
\{r: \gamma(\{\|x\| \leq r\}) \not 0 \text { or } 1\}
$$

is also an $N^{-\frac{1}{2}} O$-equivalence class.

At last, the authors would like to thank N. Cutland for kindly sending them the preprint of [2] which is crucial for the present paper. Also, they thank the referee for comments which improved the presentation of the paper.

\section{REFERENCES}

[1] N. Cutland, Infinitesimal in action, J. London Math. Soc. 35 (1987), 202-216. MR 88d:26045

[2] N. Cutland and S.-A. Ng, The Wiener sphere and Wiener measure, Annals of Probability 21 (1993), 1-13. MR 94b:03106

[3] Bang-He Li and Ji-Jiang Zhang, On the Dedekind completion of ${ }^{*} R^{*}$, Sys. Sci. \& Math. Sci. 1 (1988), 29-39. MR 90j:03113

Institute of Systems Science, Academia Sinica, Beijing 100080, People's Republic of CHINA

E-mail address: libh@iss06.iss.ac.cn

E-mail address: yli@iss06.iss.ac.cn 